\title{
ArcheoSciences
}

Revue d'archéométrie

\section{Analyse de l'organisation spatiale de deux sites de production du fer dans le Sud-Ouest du Niger}

Analysis of Spatial Organization at Two Iron-Smelting Sites in Southwestern Niger

Rodrigue Guillon, Christophe Petit, Jean-Louis Rajot, Vincent Bichet, David Sebag, Oumarou Amadou Ide et Zibo Garba

\section{QpenEdition}

\section{Journals}

\section{Édition électronique}

URL : https://journals.openedition.org/archeosciences/4015

DOI : 10.4000/archeosciences.4015

ISBN : 978-2-7535-2755-3

ISSN : $2104-3728$

Éditeur

Presses universitaires de Rennes

\section{Édition imprimée}

Date de publication : 17 avril 2013

Pagination : 123-135

ISBN : 978-2-7535-2757-7

ISSN : $1960-1360$

Référence électronique

Rodrigue Guillon, Christophe Petit, Jean-Louis Rajot, Vincent Bichet, David Sebag, Oumarou Amadou Ide et Zibo Garba, « Analyse de l'organisation spatiale de deux sites de production du fer dans le SudOuest du Niger », ArcheoSciences [En ligne], 37 | 2013, mis en ligne le 17 avril 2015, consulté le 01 février 2022. URL : http://journals.openedition.org/archeosciences/4015; DOI : https://doi.org/ 10.4000/archeosciences.4015 


\title{
Analyse de l'organisation spatiale de deux sites de production du fer dans le Sud-Ouest du Niger
}

\section{Analysis of Spatial Organization at Two Iron-Smelting Sites in Southwestern Niger}

\author{
Rodrigue Guillon*, Christophe Petit**, Jean-Louis Rajot***, \\ Vincent BICHeT ${ }^{* * * *}$, David SEBAG*****, Oumarou Amadou IDE****** \\ et Zibo GARBA ${ }^{* * * * * * *}$
}

\begin{abstract}
Résumé: Dans la région du Sud-Ouest du Niger, des bas fourneaux à usage unique ont été identifiés lors d'une prospection archéologique. À Saga Gorou, dans les environs de Niamey, un inventaire exhaustif de 4504 bas fourneaux permet de proposer une analyse spatiale au sein d'un bassin versant, de $32 \mathrm{~km}^{2}$, représentatif du paysage sahélien. Les sites métallurgiques de Bilfouda et de Garbel ont les plus fortes concentrations de bas fourneaux. Chacun d'eux a été cartographié avec précision à partir de photographies aériennes à basse altitude. Six dates radiocarbone, obtenues sur ces sites, sont comprises entre le $\mathrm{IV}^{\mathrm{e}}$ et le $\mathrm{IX}^{\mathrm{e}}$ siècles. Une analyse spatiale à partir d'outils mathématiques (classification ascendante hiérarchique, k-moyennes, arbre couvrant de poids minimum) a mis en évidence des regroupements imbriqués de bas fourneaux. Quatre niveaux d'organisation, du local au plus global, ont été identifiés: la batterie, l'ensemble, le site, et le complexe. Chacun est défini par un nombre de bas fourneaux, une superficie, une durée de fonctionnement et un facteur déterminant le contrôle de la production. Au sein de la batterie, les bas fourneaux sont disposés en forme de fer à cheval ou en lignes plus ou moins rectilignes. Cette disposition est liée à une gestion maîtrisée de l'espace de travail et semble régie par des rituels socioculturels dépendant de la famille ethnique des métallurgistes.
\end{abstract}

Abstract: In southwestern Niger, single-use furnaces were identified during archaeological prospecting. An exhaustive inventory of 4,504 bloomery furnaces from Saga Gorou, near Niamey, allowed us to propose a spatial analysis of catchment area of $32 \mathrm{~km}^{2}$, representing the Sahelian landscape. The smelting sites of Bilfouda and Garbel have the highest concentrations of bloomery furnaces; each was mapped accurately from low-altitude aerial photographs. Six radiocarbon dates were obtained, ranging from the fourth to the ninth century. A spatial analysis with a series of mathematical tools (Ward's method, $k$-means and minimum spanning tree) showed an organization of bloomery furnaces where groups were nested. Four levels from local to global were identified: battery, cluster, site and complex. Each was defined by a number of bloomery furnaces, an area, an operating time and control of production parameters. In a battery, bloomery furnaces can be arranged in a horse-shoe shape or in a more or less straight line. This organization of bloomery furnaces could be linked to the socio-cultural context and reflects good workspace management.

Mots-clés: Afrique, bas fourneaux, espace de travail, métallurgie du fer, organisation spatiale, Sahel, ${ }^{\text {er }}$ millénaire AD.

Keywords: Africa, bloomery furnaces, iron smelting, Sahel, spatial organization, workspace, 1st millennium AD.

\footnotetext{
*ArTeHiS, UMR 6298, Dijon, France. Correspondant (rodrigue.guillon@u-bourgogne.fr)

**ArScAn, UMR 7041, Paris1-Panthéon-Sorbonne, France. (christophe.petit@univ-paris1.fr)

*** Bioemco, UMR 211, IRD, Paris Est Créteil, France. (jeanlouis.rajot@ird.fr)

****Chrono-environnement, UMR 6249, Besançon, France. (vincent.bichet@univ-fcomte.fr)

*****M2C, UMR 6143, Mont-Saint-Aignan, France (david.sebag@univ-rouen.fr); HSM, UMR 5569, IRD, Montpellier, France.

******IRSH, UAM, Niamey, Niger. (oumarou@refer.ne)

******AIRE-Développement, UAM, Niamey, Niger. (zibo_garba@yahoo.com)
} 


\section{INTRODUCTION}

En Afrique subsaharienne, la métallurgie du fer est attestée à partir du $\mathrm{I}^{\mathrm{er}}$ millénaire avant notre ère (Clist, 2012). La production de fer, métal d'intérêt socio-économique majeur, s'est par la suite généralisée sur l'ensemble des régions pourvues de minerai. Les pratiques métallurgiques se sont diversifiées et intensifiées au cours du $\mathrm{I}^{\text {er }}$ millénaire de notre ère. Dans la région de Niamey (Niger), les sites archéologiques de production du fer sont identifiés à la surface du sol par la présence d'une multitude de blocs de scories ou de fonds de cuve dispersés. Ils indiquent, selon l'état d'érosion du sol, la présence d'un grand nombre de bases de fourneaux à usage unique, c'est-à-dire qu'un four est construit pour chaque opération de réduction. La répartition de ces bas fourneaux montre ainsi des regroupements où se concentre l'activité métallurgique. Cette configuration révèle une méthode de réduction du minerai de fer encore peu connue et reconnue en Afrique de l'Ouest. Elle se distingue nettement des grands sites sidérurgiques constitués de monticules de scories, correspondant à l'accumulation des déchets de réduction, produits par des bas fourneaux à usages multiples (de Barros, 1986, 2000; Huysecom et al., 2004; Robion-Brunner, 2010). Des structures à usage unique ont, cependant, déjà été cartographiées dans la région sahélienne burkinabè (Fabre, 2009) et sur les rives du Sénégal en Mauritanie (Robert-Chaleix, 1994; Robert-Chaleix et Sognane, 1983). Au Sud-Ouest du Niger, les métallurgistes ont utilisé jusqu'à la veille de la décolonisation des bas fourneaux à usage unique, montrant une pratique ritualisée; la localisation et la disposition des structures de réduction étaient soumises à des contraintes sociétales et environnementales. En effet, l'emplacement et la construction des bas fourneaux sont dépendants des familles de forgerons dont les pratiques symboliques sont nombreuses et directement liées à la technique; un couvert végétal suffisant pour fournir le combustible et du minerai de fer de qualité et en abondance sont également des éléments qui influencent le choix des métallurgistes pour implanter leur site de production du fer (Echard, 1983).

Au sein d'une zone prospectée de $32 \mathrm{~km}^{2}$ comptant 4504 bas fourneaux à usage unique, deux fortes concentrations de bas fourneaux ont été repérées et mises en évidence par une mesure de densité par la méthode du noyau (Guillon et al., 2012). Ces deux entités situées dans les localités de Bilfouda et de Garbel possèdent un grand nombre de bas fourneaux dont la distribution spatiale à toutes les échelles est agrégée. Les bas fourneaux sont principalement localisés sur les surfaces sableuses en contrebas des talus de plateaux, où affleurent les oolithes ferrugineuses qui ont été exploitées comme minerai de fer (Guillon et al., 2012).

L'objectif de cette étude est de mettre en évidence, au sein de ces deux concentrations, l'organisation des bas fourneaux à différentes échelles spatiales en recherchant l'influence de facteurs environnementaux, chronologiques et humains. Pour cela, plusieurs méthodes mathématiques provenant de l'analyse spatiale ont été utilisées.

\section{ConTEXTE GÉNÉRAL}

Les concentrations de bas fourneaux localisées près des villages de Bilfouda et de Garbel sont situées en zone climatique sahélienne, à $20 \mathrm{~km}$ à l'est de Niamey, dans le bassin versant du lac de Bangou Kirey appartenant au complexe limnique de Saga Gorou (figure 1). Ce lac est installé dans le lit d'une paléo-rivière asséchée appelée Kori de Ouallam, un ancien affluent en rive gauche du fleuve Niger. Le bassin versant est drainé par de larges ravines qui incisent le bas glacis sableux en partie cultivé. Le paysage steppique est principalement marqué par les plateaux qui dominent d'une cinquantaine de mètres le bas-fond.

Surplombant les sites métallurgiques, les talus de plateau constitués en partie de bancs d'oolithes ferrugineuses ont été exploités pour extraire le minerai de fer, comme en témoignent les blocs d'oolithes concassés retrouvés en tas à proximité des fours et ceux non fondus observés dans certaines cuves où la réduction a été incomplète. Les bas fourneaux sont tous à usage unique, mais selon la morphologie des scories nous distinguons nettement trois types pour lesquels nous présentons une description succincte. Le type I est identifié par des scories piégées en forme d'anneau dont le volume est d'environ $0,1 \mathrm{~m}^{3}$. Le type II est constitué de scories coulées accumulées formant un cylindre d'environ $0,1 \mathrm{~m}^{3}$. Le type III est formé de scories coulées de faible volume (environ $0,01 \mathrm{~m}^{3}$ ). Ces bas fourneaux appartiennent à un large secteur géographique où plusieurs autres types de bas fourneaux existent, cependant seule la région de Saga Gorou a été recensée de manière exhaustive.

Le groupe de Bilfouda est situé au centre du bassin versant de Bangou Kirey. Il se compose de 470 bas fourneaux de même typologie (type II) qui, selon les datations radiocarbone de deux structures de réduction, ont fonctionné entre le $\mathrm{IV}^{\mathrm{e}}$ et le $\mathrm{VI}^{\mathrm{e}}$ siècles $\mathrm{AD}$ (tableau 1). Quant au groupe de Garbel, situé sur la bordure sud du bassin versant à $1800 \mathrm{~m}$ de Bilfouda, il est constitué de 456 bas fourneaux des types I, II et III; quatre bas fourneaux ont été datés par radiocarbone entre le $\mathrm{VII}^{\mathrm{e}}$ et le $\mathrm{Ix}^{\mathrm{e}}$ siècles $\mathrm{AD}$ (tableau 1). 

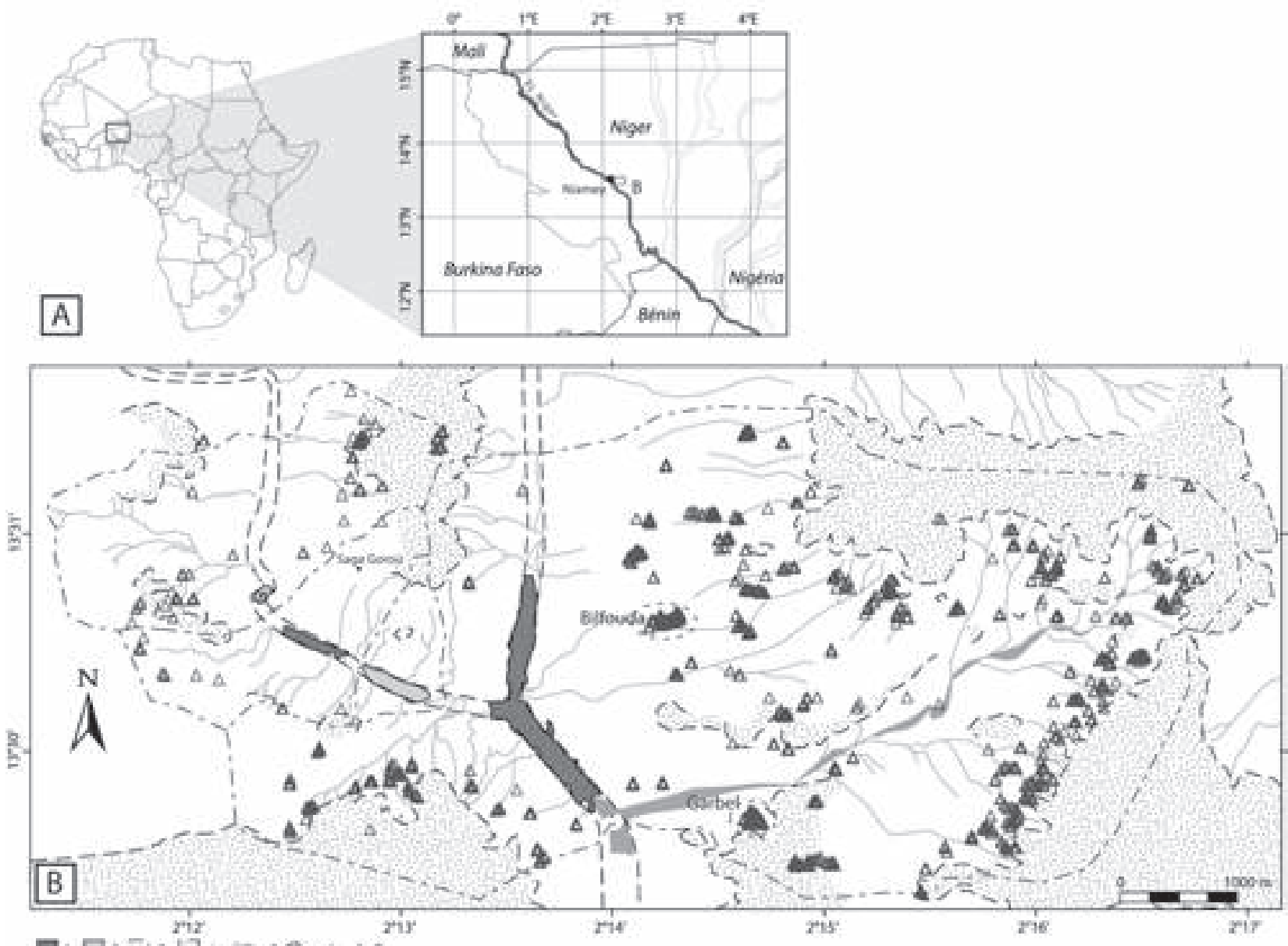

Figure 1: Localisation du site d'étude.

Figure 1: Location maps.

A- Le Sud-Ouest du Niger. B- Le bassin versant de Saga Gorou. 1 : lac permanent; 2: lac temporaire; 3 : limite de bassin versant; 4: plateaux et haut et moyen glacis; 5 : ravines; 6 : réseau fluviatile ancien; 7 : bas fourneau ( 1 triangle $=1$ bas fourneau).

A-Southwestern Niger. B-Catchment area of Saga Gorou. 1: permanent lake; 2: temporary lake; 3: catchment area boundary; 4: plateaus, haut glacis and middle glacis; 5: gullies; 6: ancient river; 7: bloomery furnace (1 triangle $=1$ furnace).

\begin{tabular}{|c|c|c|c|c|c|c|}
\hline Nom & Type de bas fourneaux & Type de fours & Matériel & Code Labo & Date ${ }^{14}$ C BP & Date cal AD $(2 \sigma)$ \\
\hline BFD_02 & Bilfouda & II & Charbon de bois & Ly-6321 & $1625 \pm 30$ & $352-537$ \\
\hline BFD_01 & Bilfouda & II & Charbon de bois & Ly-6320 & $1600 \pm 30$ & $404-540$ \\
\hline GRB_14 & Garbel & I & Charbon de bois & Ly-6333 & $1375 \pm 30$ & $607-685$ \\
\hline GRB_16 & Garbel & II & Charbon de bois & Ly-6335 & $1310 \pm 30$ & $656-773$ \\
\hline GRB_11 & Garbel & III & Charbon de bois & Ly-6331 & $1290 \pm 30$ & $663-775$ \\
\hline GRB_15 & Garbel & II & Charbon de bois & Ly-6334 & $1225 \pm 30$ & $690-885$ \\
\hline
\end{tabular}

Tableau 1: Datations radiocarbone de six bas fourneaux. La localisation des bas fourneaux datés est précisée sur les figures 3 et 6 . (Calibration Oxcal 4.1, courbe IntCal 09; Bronk Ramsey, 2009).

Table 1: Radiocarbon dating of six bloomery furnaces. Location of dated furnaces is shown in figures 3 and 6. (Calibration Oxcal 4.1, curve IntCal 09; Bronk Ramsey, 2009). 


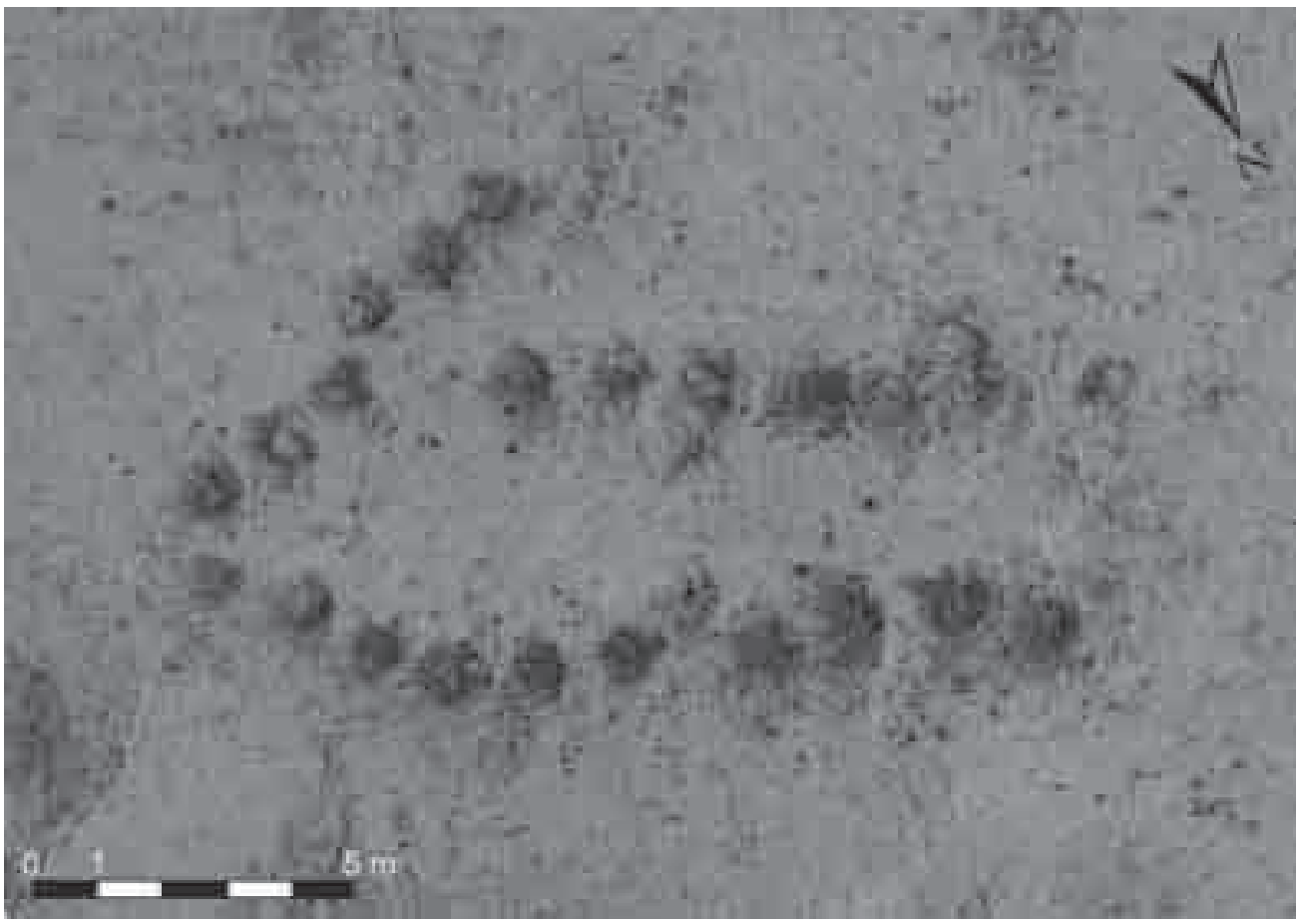

Figure 2: Photographie aérienne à basse altitude de batteries de bas fourneaux en ligne du site métallurgique de Garbel (1329’40,45”N - 2¹4’40,80”E).

Figure 2: Low-altitude aerial photography of batteries of bloomery furnaces disposed in a line from smelting site of Garbel (1329'40,45”N-2\%14'40,80"E).

(C) JL Rajot)

\section{Méthodes}

Les bas fourneaux ont été cartographiés à partir de photographies aériennes à basse altitude (figure 2) qui ont été géoréférencées et orthorectifiées à l'aide d'un Système d'Information Géographique (SIG). Le relevé des structures de réduction a été complété par une seconde mission sur le terrain afin de corriger et de compléter le plan sur les zones où les bas fourneaux étaient peu visibles. La précision de la position géographique des bas fourneaux est de l'ordre du mètre.

La distribution spatiale agrégée des bas fourneaux ouvre la possibilité de réaliser plusieurs analyses spatiales dans le but de caractériser et d'individualiser des groupes; pour cela différentes méthodes ont été employées sous SIG (ArcGIS) et avec le logiciel $\mathrm{R}$.

\section{La classification ascendante hiérarchique selon la méthode de Ward}

Une Classification Ascendante Hiérarchique (CAH) a pour but de partitionner en classes distinctes un nuage de points. Pour cela, la distance euclidienne entre les points est calculée; elle correspond à la distance la plus courte entre deux éléments.

La distance entre classes est, quant à elle, calculée selon la méthode de Ward en utilisant une analyse de la variance. Cette méthode définit alors une arborescence telle que la somme des distances au carré entre les points et le centre des groupes auxquels ils appartiennent soit minimale (Ward, 1963). Cette méthode fournit une hiérarchie de partitions, les groupes les plus significatifs sont déterminés par coupure de l'arbre en fonction des valeurs de dissimilarité (Lebart et al., 2000).

\section{La Méthode des k-moyennes}

Cette méthode de partition, fondée sur la distance entre points, produit une répartition en secteurs, ici des ellipses, pour lesquels $\mathrm{K}$ barycentres sont déterminés de manière arbitraire dans un nuage de points (Zaninetti, 2005). Elle permet ainsi de diviser ce nuage en un nombre donné de groupes dont le noyau est défini par un critère de séparation. 
Les centres des groupes sont alors recalculés pour chaque point ajouté, si un point appartenant à un groupe se trouve plus proche du centre d'un autre groupe, il est réattribué (Conolly \& Lake, 2006). Cette méthode ne permet pas de discriminer les groupes organisés en ligne.

\section{L'arbre couvrant de poids minimum}

Dans un nuage de points quelconque, la représentation de l'arbre couvrant de poids minimum est celle d'un graphe connexe et sans cycle pour lequel la somme des distances entre les points est minimale (Gower et Ross, 1969; Zahn, 1971). Cette méthode permet d'établir un lien de proche en proche entre les bas fourneaux en recherchant la plus courte distance entre les individus, ce qui permettra pour notre jeu de données de mettre en évidence un plan d'organisation.

\section{RÉSUlTATS}

\section{Le site de Bilfouda}

Le dendrogramme issu de la classification ascendante hiérarchique selon la méthode de Ward appliquée à l'ensemble des bas fourneaux, tous de type II, fournit quatre groupes
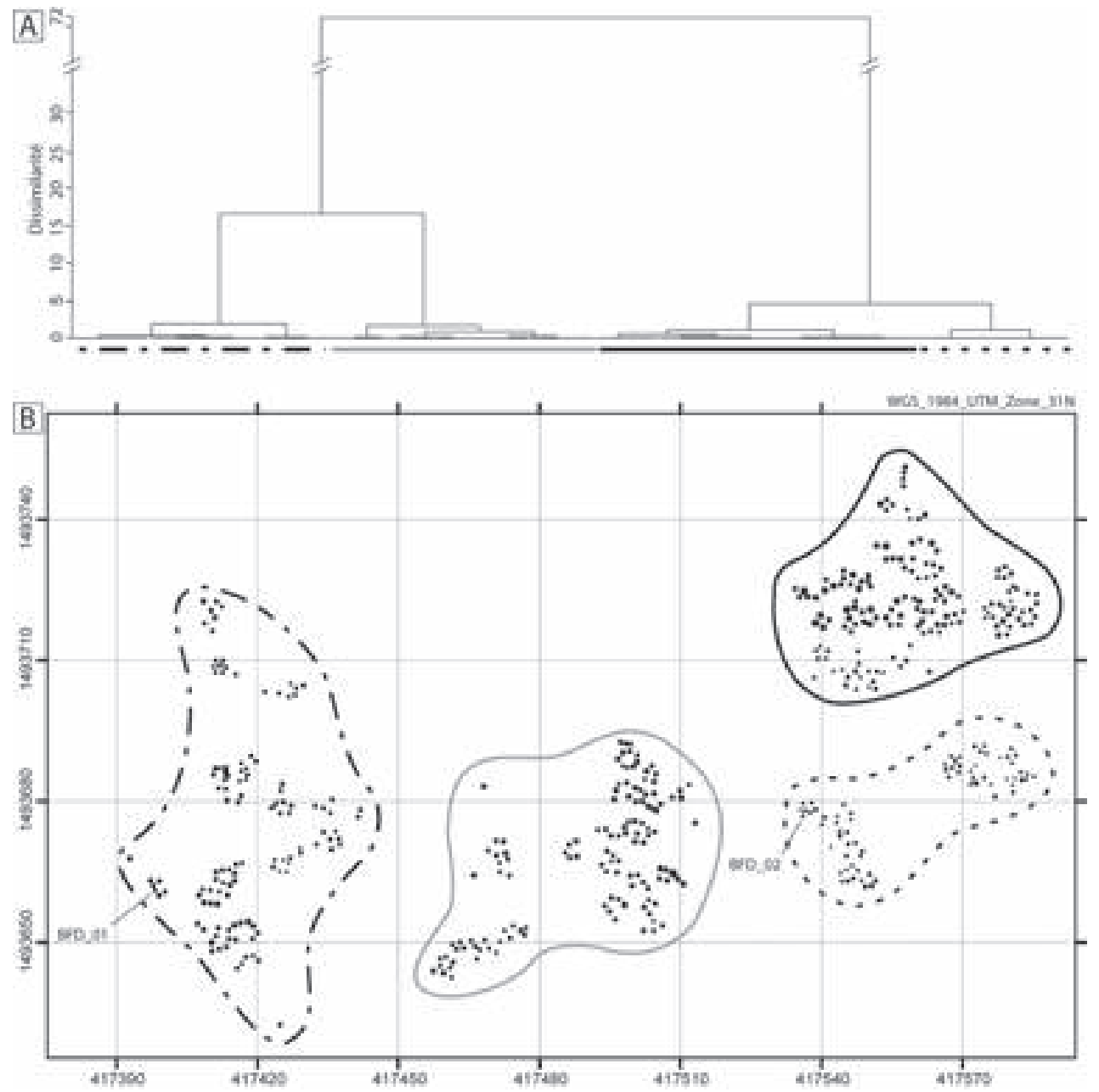

Figure 3: Le site métallurgique de Bilfouda.

Figure 3: Smelting site of Bilfouda.

A- Dendrogramme de Ward. B- Carte de répartition des bas fourneaux (type II) et les quatre groupes principaux issus de la CAH selon la méthode de Ward.

A- Ward's dendrogram. B-Distribution map of bloomery furnaces (type 2) and four Ward's method groups. 


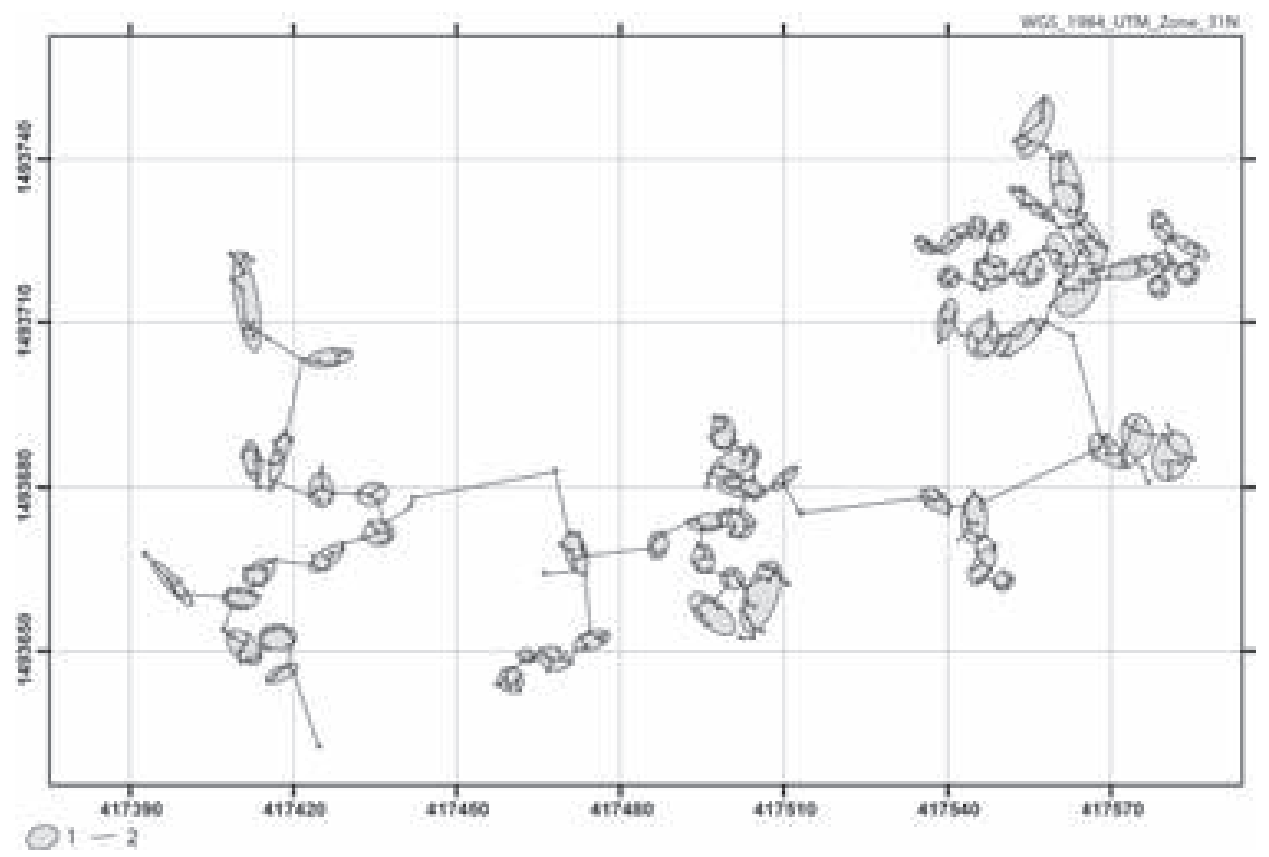

Figure 4: Le Site métallurgique de Bilfouda.

Figure 4: Smelting site of Bilfouda.

1 : groupes issus de la méthode des k-moyennes ; 2 : arbre couvrant de poids minimum.

$1: k$-means groups $; 2$ : minimum spanning tree.

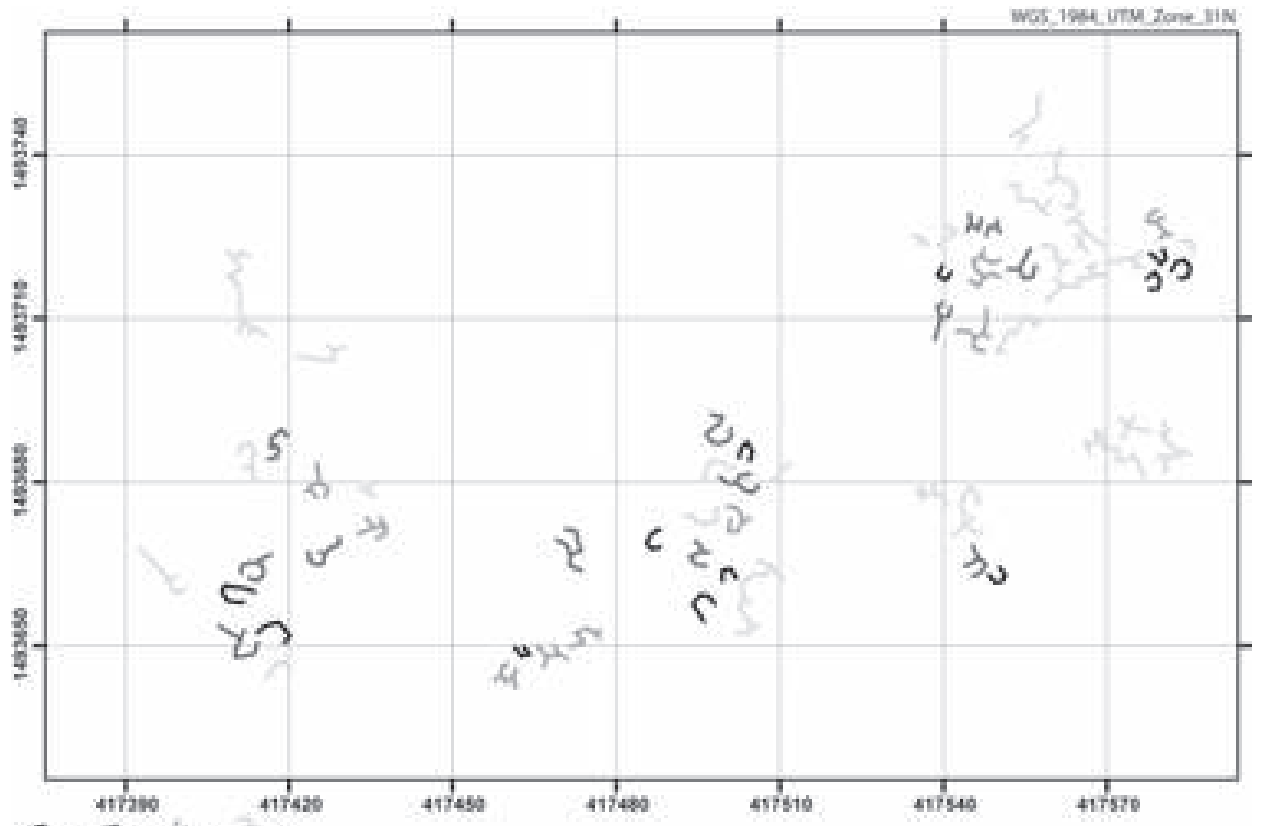

$C_{1}<_{2}<3<4$

Figure 5: L'organisation des bas fourneaux au sein du site métallurgique de Bilfouda. Figure 5: Organization of bloomery furnaces in smelting site of Bilfouda.

1 : forme en $\mathrm{C}$; 2 : forme en $\mathrm{C}$ avec une extension; 3 : forme en $\mathrm{C}$ avec deux extensions ; 4 : forme complexe.

1: C-shape; 2: C-shape with an extension; 3: C-shape with two extensions; 4: complex shape. 
principaux (figure 3-A). Ils sont constitués respectivement de 70, 121, 126 et 153 bas fourneaux occupant chacun une superficie d'environ $1000 \mathrm{~m}^{2}$ (figure 3-B). Les groupes de niveau inférieur ne peuvent pas être individualisés par cette méthode étant donné les faibles valeurs de dissimilarité; une méthode de partition est alors nécessaire.

La méthode des k-moyennes a permis de déterminer des groupes de plus petites dimensions en recherchant le nombre maximal de groupes parmi l'ensemble des structures de réduction. 59 groupes sont ainsi mis en évidence (figure 4), ils sont matérialisés par des ellipses qui symbolisent les surfaces sur lesquelles est regroupé le plus petit nombre de bas fourneaux possible. La superficie de ces ellipses est comprise entre 6 et $63 \mathrm{~m}^{2}$ avec une valeur médiane de $19 \mathrm{~m}^{2}$. Le nombre de bas fourneaux par ellipse est compris entre 4 et 15 , avec une médiane de 7 qui représente près d'un quart des ellipses identifiées; $90 \%$ de ces groupes contiennent moins de 11 bas fourneaux.

L'arbre recouvrant de poids minimum permet d'établir une relation spatiale entre les bas fourneaux. Seules les relations matérialisées à l'intérieur de chaque ellipse définie par la méthode des k-moyennes ont été considérées (figure 4). Nous obtenons ainsi un réseau dessinant un plan d'organisation plus ou moins complexe dont la forme de base rappelle celle d'un fer à cheval ou de la lettre C; 34 entités ont été identifiées (figure 5). Nous distinguons ainsi 12 entités dont le plan correspond à la forme d'un fer à cheval (forme en C simple), 13 ayant une forme en $\mathrm{C}$ à laquelle est ajouté en périphérie un ou deux bas fourneau(x), ce qui crée une extension, et neuf comportant deux extensions composées de plus de deux bas fourneaux. Pour le reste, les outils mathématiques ne proposent pas de modèles simples.

\section{Le site de Garbel}

À Garbel, où trois types de bas fourneaux coexistent, la $\mathrm{CAH}$ selon la méthode de Ward a été appliquée à l'ensemble des bas fourneaux sans distinction typologique. Nous pouvons ainsi déterminer selon les classes du dendrogramme trois groupes de 60, 166 et 230 bas fourneaux (figure 6). Chacun d'eux contient les trois types de bas fourneaux ce qui sous-entend qu'à cette échelle d'observation, la typologie des bas fourneaux est indépendante de la répartition spatiale. Toutefois, certains sous-groupes isolés sont constitués de bas fourneaux identiques.

Pour chacune des trois typologies, l'arbre couvrant de poids minimum permet de mettre en évidence des alignements de bas fourneaux. Ces lignes rectilignes et sinueuses rassemblent $42 \%$ des bas fourneaux en 34 groupes (figure 7). Chaque ligne est composée de trois à dix bas fourneaux de typologie identique, certaines sont disposées en parallèle, en chevron ou en épingle. Parmi les 169 bas fourneaux disposés en lignes, 2/3 sont de type I. Quant aux bas fourneaux de type II, ils forment des lignes plus sinueuses, d'autres ont une disposition plus difficile à définir qui s'apparente à des cercles ou à des fers à cheval, comme celle décelée à Bilfouda (figure 6). Pour le type III, aucune ligne ni autre organisation n'est repérée. Chaque type est ainsi caractérisé par un ou plusieurs plans d'organisation.

\section{Discussions}

\section{Organisation spatiale multiscalaire}

L'utilisation d'outils mathématiques permet de mettre en évidence l'existence de concentrations de bas fourneaux à différentes échelles spatiales. La localisation et la disposition de ces bas fourneaux correspondent à un choix qui émane de la décision du métallurgiste pour répondre au protocole technique et aux contraintes sociétales (ethniques) et rituelles qu'il s'impose. Les différents regroupements correspondent à des entités géographiques dont la nomenclature n'est pas clairement établie; nous nous contenterons alors d'utiliser des termes explicites pour les quatre niveaux de regroupements repérés, du plus global au local, soit: complexe, site, ensemble et batterie (tableau 2).

Le groupe le plus large constitue un complexe estimé à plusieurs millions de bas fourneaux et couvrant une superficie de plus de $10000 \mathrm{~km}^{2}$. Plusieurs siècles sont nécessaires à son élaboration et il se définit par une tradition métallurgique qui se caractérise par la construction de bas fourneaux à usage unique.

Au sein de ce complexe, une multitude de sites comme ceux de Bilfouda et de Garbel sont identifiés. Ils comptent environ 500 bas fourneaux qui se répartissent sur une superficie comprise entre un et cinq hectare(s). Leur fonctionnement, d'après les dates radiocarbone, s'étend sur plusieurs décennies voire quelques siècles et leur organisation est déterminée par des facteurs géomorphologiques (minerai, nature du sol) (Guillon et al., 2012) et anthropologiques (sociétal et territorial).

À l'intérieur de ces sites, des ensembles peuvent être délimités, ils regroupent entre 50 et 200 bas fourneaux qui se dispersent sur une surface comprise entre 1000 et $4000 \mathrm{~m}^{2}$. Leur conception est issue soit d'une main-d'œuvre nombreuse sur un temps limité, telle une saison ou une année, soit d'un groupe restreint de personnes sur un temps long (quelques décennies). Ces ensembles peuvent avoir, comme à Garbel, la particularité de contenir des bas fourneaux de 


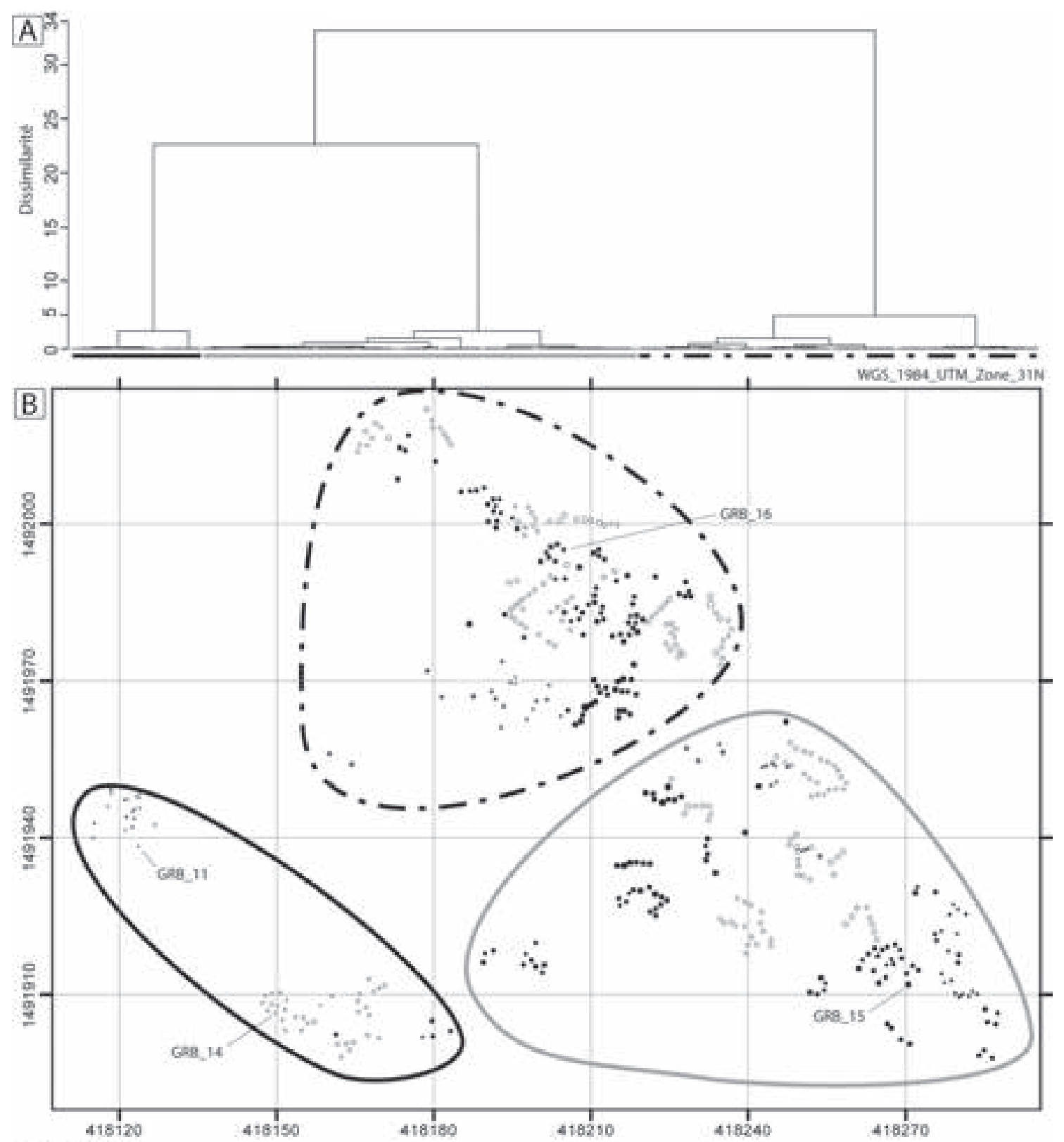

Figure 6 : Le site métallurgique de Garbel.

Figure 6: Smelting site of Garbel.

A- Dendrogramme de Ward. B- Carte de répartition des bas fourneaux et les trois groupes issus de la CAH selon la méthode de Ward. 1 : bas fourneaux de type I; 2 : bas fourneaux de type II ; 3 : bas fourneaux de type III.

A-Ward's dendrogram. B-Distribution map of bloomery furnaces and three Ward's method groups. 1: bloomery furnace type 1; 2: bloomery furnace type 2; 3: bloomery furnace type 3. 


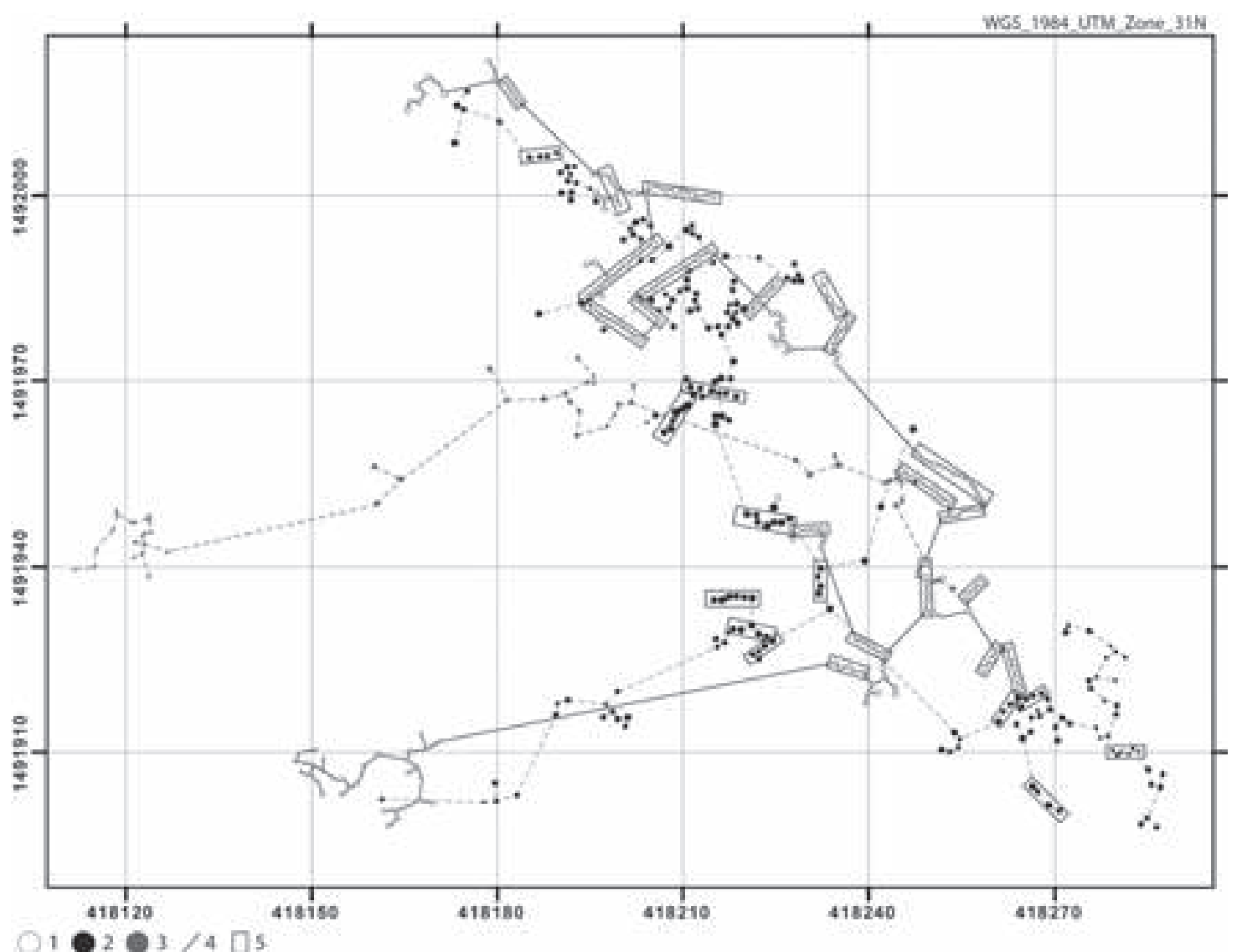

Figure 7: Organisation des bas fourneaux du site métallurgique de Garbel.

Figure 7: Organization of bloomery furnaces in smelting site of Garbel.

1 : bas fourneaux de type I; 2 : bas fourneaux de type II; 3: bas fourneaux de type III ; 4 : arbre couvrant de poids minimum ; 5 : batterie disposée en ligne.

1: bloomery furnace type 1; 2: bloomery furnace type 2; 3: bloomery furnace type 3; 4: minimum spanning tree; 5: battery disposed in line.

typologies différentes. Cela souligne l'indépendance des groupes de métallurgistes et de leur pratique par rapport au lieu d'implantation des bas fourneaux.

Ces ensembles sont composés de batteries de bas fourneaux. Celles-ci forment le plus petit regroupement spatial et correspondent à un atelier fonctionnant pendant un temps donné. Les fours étant à usage unique, l'atelier se déplace de batterie en batterie à chaque temps de production. Elles sont constituées au plus d'une quinzaine de bas fourneaux de même typologie et couvrent entre 10 et $50 \mathrm{~m}^{2}$. Leur mise en place, qui nécessite quelques jours, peut être issue de la coordination de métallurgistes pouvant appartenir à des groupes familiaux ou à une coopération. En effet, dans l'Ader près de la frontière du Nigéria, à $300 \mathrm{~km}$ à l'est du site d'étude, N. Echard (1983) décrit de telles concentrations de bas fourneaux datant de la fin du XIX et du début du $\mathrm{XX}^{\mathrm{e}}$ siècles, où cette coopération désigne un regroupement de métallurgistes provenant d'un même village ou d'une même région.

L'unité de base que constitue le bas fourneau fonctionne sur une journée (environ 24 heures) et occupe un espace de près d'un mètre carré, il dépend de paramètres technique et anthropologique (rituel) qui sont indissociables (Echard, 1983; Huysecom, 2001).

L'organisation des bas fourneaux est donc régie par divers paramètres, géomorphologiques, techniques et anthropologiques qui diffèrent à chacune des échelles spatiales considérées. Chaque niveau de regroupement reflète par ailleurs des durées de fonctionnement qui s'échelonnent de la journée à plusieurs siècles (tableau 2). 


\begin{tabular}{|c|c|c|c|c|}
\hline Nomenclature & Nombre de bas fourneaux & Surface & Durée de fonctionnement & Contrôle de la production \\
\hline Complexe & $>$ à 1 million & $>$ à $10000 \mathrm{~km}^{2}$ & Siècles & Tradition métallurgique \\
\hline Site & Environ 500 & 1 à $5 \mathrm{ha}$ & Décennies/siècle & $\begin{array}{c}\text { Géomorphologique et anthropologique } \\
\text { (sociétal, territorial) }\end{array}$ \\
\hline Ensemble & Entre 50 et 250 & 1000 à $4000 \mathrm{~m}^{2}$ & Année/décennies & Intensité et/ou chronologie \\
\hline Batterie & $<$ à 15 & 10 à $50 \mathrm{~m}^{2}$ & Quelques jours & Famille et/ou Coopération \\
\hline Bas fourneau & Un & $1 \mathrm{~m}^{2}$ & Un jour & Technique et anthropologique (rituel) \\
\hline
\end{tabular}

Tableau 2 : Terminologie, définition et interprétation de chaque niveau de regroupement. Table 2: Terminology, definition and interpretation of each level of clustering.

Si l'on observe des organisations spécifiques à différentes échelles spatiales, seule l'organisation des batteries peut faire l'objet de comparaisons ethnographiques.

\section{Organisation des batteries}

Tous les bas fourneaux ne sont pas construits au sein de batteries et celles-ci ne sont pas toutes rigoureusement organisées selon un agencement précis; si certains bas fourneaux sont disposés en ligne ou en fer à cheval, d'autres sont isolés ou regroupés sans ordre particulier. Cependant, l'agencement des batteries tel qu'il a pu être mis en évidence pour la plupart des bas fourneaux de ces sites, traduirait une organisation correspondant à un savoir-faire du métallurgiste. En effet, dans le cas d'une production simultanée et d'une disposition en fer à cheval, l'espace libre au centre des bas fourneaux paraît adapté pour contrôler l'ensemble des bas fourneaux qui aurait alors fonctionné simultanément. L'espacement plus large entre deux structures de réduction crée une ouverture facilitant la circulation entre le cœur de l'atelier et l'extérieur. Cette disposition peut alors s'apparenter à un aménagement ergonomique tout en répondant à des pratiques rituelles. Quant aux bas fourneaux présents en périphérie, ils peuvent être interprétés comme étant dévolus à une production de fer secondaire par rapport à ceux disposés en fer à cheval. Des structures de réduction disposées en ligne sont, quant à elles, connues dans l'Ader où une étude ethnographique révèle l'organisation d'ateliers de réduction du fer construits au cours de la première moitié du $\mathrm{xx}^{\mathrm{e}}$ siècle (Echard, 1983). Selon ses enquêtes, les lignes, dont le type de bas fourneaux est différent de ceux de cette étude, ont été construites suivant un axe nord/sud. Le fer produit dans le bas fourneau central était destiné au chef de l'atelier (campement du fer), les bas fourneaux placés au sud produisaient pour les parents du métallurgiste et ceux situés au nord pour des clients. À Garbel, les alignements de bas fourneaux observés ne sont pas orientés selon une direction préférentielle et il est difficile de reconnaître au sein d'un alignement une hiérarchisation.

Selon les secteurs géographiques, l'organisation des bas fourneaux prend diverses formes. Dans l'Ader (Niger) les bas fourneaux sont agencés en lignes (Echard, 1983) alors que sur les rives du Sénégal à Silla (Mauritanie) D. RobertChaleix et M. Sognane (1983) décrivent la disposition des bas fourneaux comme des cercles avec deux fours centraux ou comme de longues lignes sinueuses (figure 8). Cependant si la présence de lignes est incontestable, les plans relevés à Silla peuvent être interprétés différemment, le nuage de points pourrait être perçu entre autres comme des batteries de bas fourneaux organisées en fer à cheval comme cela est observé à Bilfouda.

En Afrique subsaharienne, l'organisation de batteries a été décrite comme étant dépendante de la typologie des bas

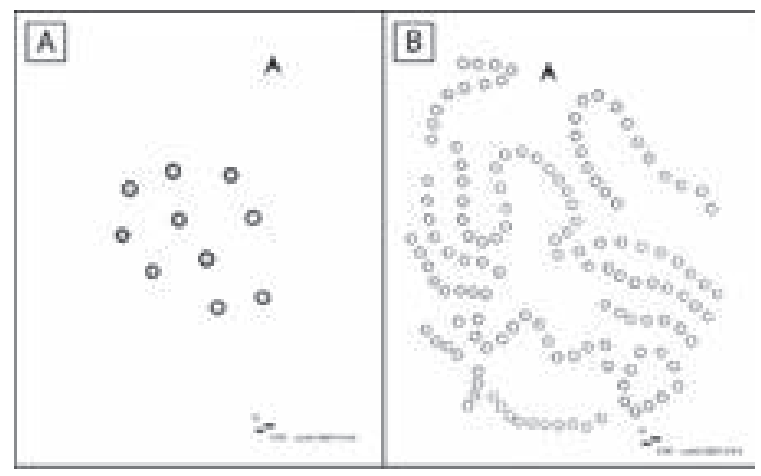

Figure 8: Bas fourneaux dans la vallée moyenne du Sénégal. Figure 8: Bloomery furnaces in the middle valley of the Senegal. A: Bas fourneaux de Silla organisés en cercle avec deux fours centraux. B: Bas fourneaux disposés en alignements sinueux (d'après Robert-Chaleix et Sognane, 1983).

A: Furnaces of Silla organized in circle with two central furnaces. B: Furnaces arranged in sinuous alignments (in Robert-Chaleix and Sognane, 1983). 
fourneaux (Gouletquer, 1983), or cette relation n'est pas systématique. En effet, à Garbel, la batterie en ligne est utilisée par les métallurgistes pour deux types de bas fourneaux différents (types I et II). De plus, en comparant les deux sites, nous constatons que les bas fourneaux de type II sont disposés en fers à cheval à Bilfouda alors qu'ils sont plutôt organisés en lignes sinueuses à Garbel. Ce changement d'organisation spatiale pour une même typologie peut être le résultat d'une évolution des pratiques au cours du temps puisque le site de Bilfouda est antérieur de quelques siècles à celui de Garbel.

Dans ce bassin versant, l'organisation de ces ateliers et la typologie des bas fourneaux sont diverses. Ces pratiques, qui paraissent techniquement indépendantes, témoigneraient alors de savoir-faire différents, propres à chaque famille de métallurgistes. Les outils mathématiques (analyses spatiale et statistique) nous ont permis de reconstituer les plans d'agencement des ateliers de métallurgie; ces regroupements de bas fourneaux reflètent potentiellement l'organisation mise en place par les métallurgistes. Les datations radiocarbone réalisées ne permettent pas de définir les différentes périodes de fonctionnement de ces ateliers, qui sont relativement courtes, de plus, elles restent trop peu nombreuses pour comprendre la chronologie de ces différentes phases d'organisation.

\section{Remerciements}

Nous tenons à remercier les relecteurs de cet article pour leurs nombreuses remarques constructives. Ce programme de recherche a été financé par le projet 6116 de la Coopération pour la Recherche Universitaire et Scientifique (CORUS2) intitulé Impacts de la pression anthropique et du changement global sur les flux sédimentaires en zone sahélienne: dynamique éolienne et aérosols terrigènes.

\section{Bibliographie}

De BArros, P., 1986. Bassar: a quantified, chronologically controlled, regional approach to a traditional iron production centre in West Africa. Africa. 56, 2: 148-174.

De Barros, P., 2000. Iron metallurgy : sociocultural context. In M. S. Bisson, S. T. Childs, P. De Barros, A. F. C. Holl (éd.). Ancient African Metallurgy, the socio-cultural context. Joseph O. Vogel: 147-198.

Bronk Ramsey, C., 2009. Bayesian analysis of radiocarbon dates. Radiocarbon, 51(1): 337-360.

Clist B., 2012. Vers une réduction des préjugés et la fonte des antagonismes: un bilan de l'expansion de la métallurgie du fer en Afrique sud-saharienne, Journal of African Archaeology, 10, 1: 71-84.

Conolly, J. et Lake, M., 2006. Geographical information systems in archaeology. Cambridge University Press, Cambridge.

Echard, N., 1983. Scories et symboles, remarques sur la métallurgie hausa du fer au Niger. In N. ECHARD (éd.). Métallurgies africaines. Nouvelles contributions, Mémoires de la Société des Africanistes, 9: 209-224.

Fabre, J. M., 2009. La métallurgie du fer au Sahel burkinabé à la fin du ${ }^{\mathrm{er}}$ millénaire AD. In S. Magnavita, L. Koté, P. Breunig, O. A. Idé (éd.). Crossroads, Cultural and technological developments in first millennium BC/AD West Africa. Journal of African Archaeology Monograph Series, 2: $167-178$.

Gouletquer, P., 1983. Territoires et technique: le sel et le fer. In N. Echard (éd.). Métallurgies africaines. Nouvelles contributions, Mémoires de la Société des Africanistes, 9: 173-197.

Gower, J. C. et Ross G. J. S., 1969. Minimum Spanning Trees and Single Linkage Cluster Analysis. Journal of the Royal Statistical Society. Series C, 18, 1: 54-64.

Guillon, R., Petit, C., Rajot, J. L., Bichet, V., Idé, O. A., Garba, Z., Sebag, D., et Abdourhamane Touré, A., 2012. Analyse de la répartition de bas fourneaux d'un territoire sidérurgique au sud-ouest du Niger (IV ${ }^{\mathrm{e}}-\mathrm{XIV}^{\mathrm{e}}$ siècle apr. J.-C.). In C. Robion-Brunner, B. Martinelli (éd.), Métallurgie du fer et Sociétés africaines : Bilans et nouveaux paradigmes dans la recherche anthropologique et archéologique, BAR International Series 2395, Cambridge Monographs in African Archaeology 81: 153-163.

Huysecom, E., 2001. Technique et croyance des forgerons africains: éléments pour une approche ethnoarchéologique. In J. P. Descoeudres, E. Huysecom, V. Serneels et Zimmermann, J.L. (éd.). Aux origines de la métallurgie du fer. Table ronde internationale d'archéologie : l'Afrique et le bassin méditerranéen (4-7 juin 1999, Genève). University of Sydney 14, Sydney: 73-82.

Huysecom, E., Ballouche, A., Cissé, L., Gallay, A., Konaté, D., Mayor, A., Neumann, K., Ozainne, S., Perret, S., Raeli, F., Rasse, M., Robert, M., Robion, C., Sanogo, K., Serneels, V., Soriano, S., Stokes, S., 2003. Paléoenvironnement et peuplement humain en Afrique de l'ouest : rapport de la sixième campagne de recherche à Ounjougou (Mali). Jahresbericht SLSA: 27-68.

Lebart, L., Morineau, A., Piron, M., 2000. Statistique exploratoire multidimensionnelle. Dunod, Paris.

Robert-Chaleix, D., 1994. Métallurgie du fer dans la moyenne vallée du Sénégal: les bas fourneaux de Silla. Journal des africanistes, 64, 2: 113-127.

Robert-Chaleix, D., Sognane, M. 1983. Une industrie métallurgique ancienne sur la rive mauritanienne du fleuve Sénégal. 
In N. EchARD (éd.). Métallurgies africaines. Nouvelles contributions, Mémoires de la Société des Africanistes, 9: 45-62.

Robion-Brunner, C., 2010. Forgerons et sidérurgie en pays dogon. Vers une histoire de la production du fer sur le plateau de Bandiagara (Mali) durant les empires précoloniaux. Journal of African Archaeology Monograph Series, 3. Africa Magna Verlag, Frankfort.

WARD, J. H., 1963. Hierarchical grouping to optimize and objective function. Journal of the American Statistical Association. 58: $238-244$.
ZAHN, C. T., 1971. Graph-theoretical methods for detecting and describing gestalt clusters. IEEE Transactions on Computers, C-20: 68-86.

Zaninetti, J.-M., 2005. Statistique spatiale : Méthodes et applications géomatiques. Lavoisier. Paris. 